\title{
Bericht aus dem Arbeitskreis Psychiatrie und Migration
}

\section{Interkulturelle Öffnung der psychiatrischen Kliniken in Deutschland}

Migration kann zu Sprach- und Verständigungsproblemen führen und Identitätsund Rollenkonflikte verstärken. Migration birgt also das Risiko persönlicher und seelischer Belastungen. Für Menschen mit Migrationserfahrung bestehen Barrieren im Gesundheitssystem, die zu einer verringerten Aufklärung über vorhandene Gesundheitsangebote und einer reduzierten Inanspruchnahme therapeutischer und präventiver Maßnahmen führen [1].

Der BDK-Arbeitskreis Migration beschäftigt sich seit dem Jahr 2001 mit der klinischen Versorgung von Patient*innen mit Migrationshintergrund. Aktuell geht es vor allem um Konzepte zur interkulturellen Öffnung der Kliniken, insbesondere die Organisation von Dolmetschern für den psychiatrischen Alltag. Auch werden kulturell angepasste Therapieangebote entwickelt und evaluiert, wie beispielsweise das Psychoedukationsangebot für Patienten mit Migrationshintergrund.

Auf der Grundlage etablierter Manuale für Psychoedukation (z. B. [2]) wurde ein migrationsbezogenes, kultursensibles Manual erarbeitet. Das „Manual Interkulturelle Psychoedukation für Menschen mit Migrationshintergrund“ [3] wird derzeit in mehreren deutschen Kliniken (u.a. LWL-Klinik Dortmund, einige Vitos-Kliniken) evaluiert. Mit fünf themenspezifischen Modulen wird das Ziel verfolgt, die Versorgungsqualität für Menschen mit Migrationserfahrung zu verbessern. Dabei steht die Wissensvermittlung über das Gesundheitssystem, die Themen Prävention und Rückfallschutz, Integration, soziale Kontakte sowie Hilfsangebote, zugleich die Vermittlung von Kenntnissen über psychische Erkrankungen im Vorder- grund. Den Themen wird ein Modul vorangestellt, das den Austausch über die Erfahrungen der Teilnehmer*innen bezüglich des Migrationsprozesses ermöglicht und die soziale Integration fördert. Die im Manual enthaltenen Arbeitsblätter sind in den Sprachen Deutsch, Arabisch und Türkisch verfügbar, und sollen helfen, die Sprachbarrieren zu vermindern.

Es wird den Fragen nachgegangen, ob es durch die Teilnahme an der Psychoedukation eine Steigerung der Behandlungszufriedenheit gibt und inwiefern die Teilnehmer*innen einen Nutzen im Alltag erfahren. Dazu dient eine Befragung mit evaluierten Fragebögen: Migrationsstressoren (MIGSTR10), Skala der allgemeinen Selbstwirksamkeit (SWE), Personal and Social Performance Scale (PSP). Die Psychoedukationsgruppen finden einmal in der Woche über 60 min statt; der Einsatz von Dolmetschern ist möglich. Die Zusammensetzung der Gruppen ist heterogen in Bezug auf das Geschlecht, die Sprache und die Diagnose(n). Pro Teilnehmer*in sind bis zu 5 Sitzungen vorgesehen.

Bisher haben 50 Patient*innen (weiblich $\mathrm{n}=27$; männlich $\mathrm{n}=23$ ) im Alter von 1869 Jahren $(M=42,6 ; S D=12,9)$ an der Evaluation teilgenommen. Die Aufenthaltsdauer in Deutschland lag zum Beginn der Psychoedukation zwischen 0-48 Jahren $(M=16,5 ; \quad S D=14,9)$. Mehrheitlich kamen die Patienten aus der Türkei (36\%). Die Muttersprache war mehrheitlich Türkisch (34\%) und Arabisch (18\%). Im Prä-Post-Vergleich ergaben sich signifikante Verbesserungen in puncto „Offenheit über Probleme zu sprechen“ ( $p=0,008)$, „Kenntnisstand“ $(p<0,001)$ sowie „Selbsteinschätzung der Sprachkompetenz“ $(p<0,001)$. Die „Zufriedenheit mit dem Angebot" war hoch (im
Durchschnitt $m=53,4$; SD=7,0 Punkte, bei einer maximalen Punktzahl von 60 Punkten).

Das Studienprojekt ist noch nicht abgeschlossen und es erfolgt derzeit der Vergleich zu einer Kontrollgruppe, einer „ungeleiteten“ Gesprächsgruppe. Durch die Praxis und die Studienerfahrungen werden Anregungen gesammelt, um eine Optimierung und Differenzierung der Psychoedukation zu erreichen. Sofern es gelingt, Menschen anderer Herkünfte zu unterstützen, Kenntnisse, Gleichberechtigung und Zufriedenheit zu fördern, hat das Projekt sein Ziel erreicht.

\section{Literatur}

[1] Koch E. Psychiatrische Versorgung und interkulturelle Öffnung. In: Graef-Calliess IT, Schouler-Ocak M, Hrsg. Migration und Transkulturalität. Stuttgart: Schattauer; 2017: 85-96

[2] Bäuml ], Behrendt B, Henningsen P, PitschelWalz G, Hrsg. Handbuch der Psychoedukation. Stuttgart: Schattauer; 2016

[3] Assion H], Ueberberg B, Kaaz T. Manual interkulturelle Psychoedukation für Menschen mit Migrationserfahrung. Online: Arbeitsmaterialien auf Türkisch und Arabisch. Stuttgart: Schattauer; 2018

\section{Korrespondenzadressen}

Prof. Dr. Eckhardt Koch

Sprecher AK Migration der BDK, Migrationsbeauftragter Vitos Holding, Kassel eckhardt.koch@vitos.de

Prof. Dr. Hans-Jörg Assion Ärztlicher Direktor LWL-Klinik Dortmund hans-joerg.assion@lwl.org 